\begin{tabular}{|c|c|c|}
\hline & Int.J.Curr.Microbiol.App.Sci (2016) 5(10): 749-758 & \multirow{2}{*}{ 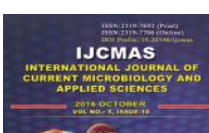 } \\
\hline & \multirow{4}{*}{$\begin{array}{l}\text { International Journal of Current Microbiology and Applied Sciences } \\
\text { ISSN: 2319-7706 Volume } 5 \text { Number } 10 \text { (2016) pp. 749-758 } \\
\text { Journal homepage: http://www.ijcmas.com }\end{array}$} & \\
\hline & & \\
\hline EXCELLENT & & \\
\hline PUBLISHERS & & wwwi.jicmas.com \\
\hline
\end{tabular}

Original Research Article

http://dx.doi.org/10.20546/ijcmas.2016.510.081

\title{
Phenotypic Characterization of ESBL, AmpC and MBL Producers among the Clinical Isolates of Multidrug Resistant Pseudomonas aeruginosa
}

\author{
Mohammed Ansar Qureshi ${ }^{1 *}$ and Rakesh Kumar Bhatnagar ${ }^{2}$
}

\author{
${ }^{1}$ Department of Microbiology, Himalayan University 791110 Itanagar India \\ ${ }^{2}$ Department of Pathology, Himalayan University 791110 Itanagar, India \\ *Corresponding author
}

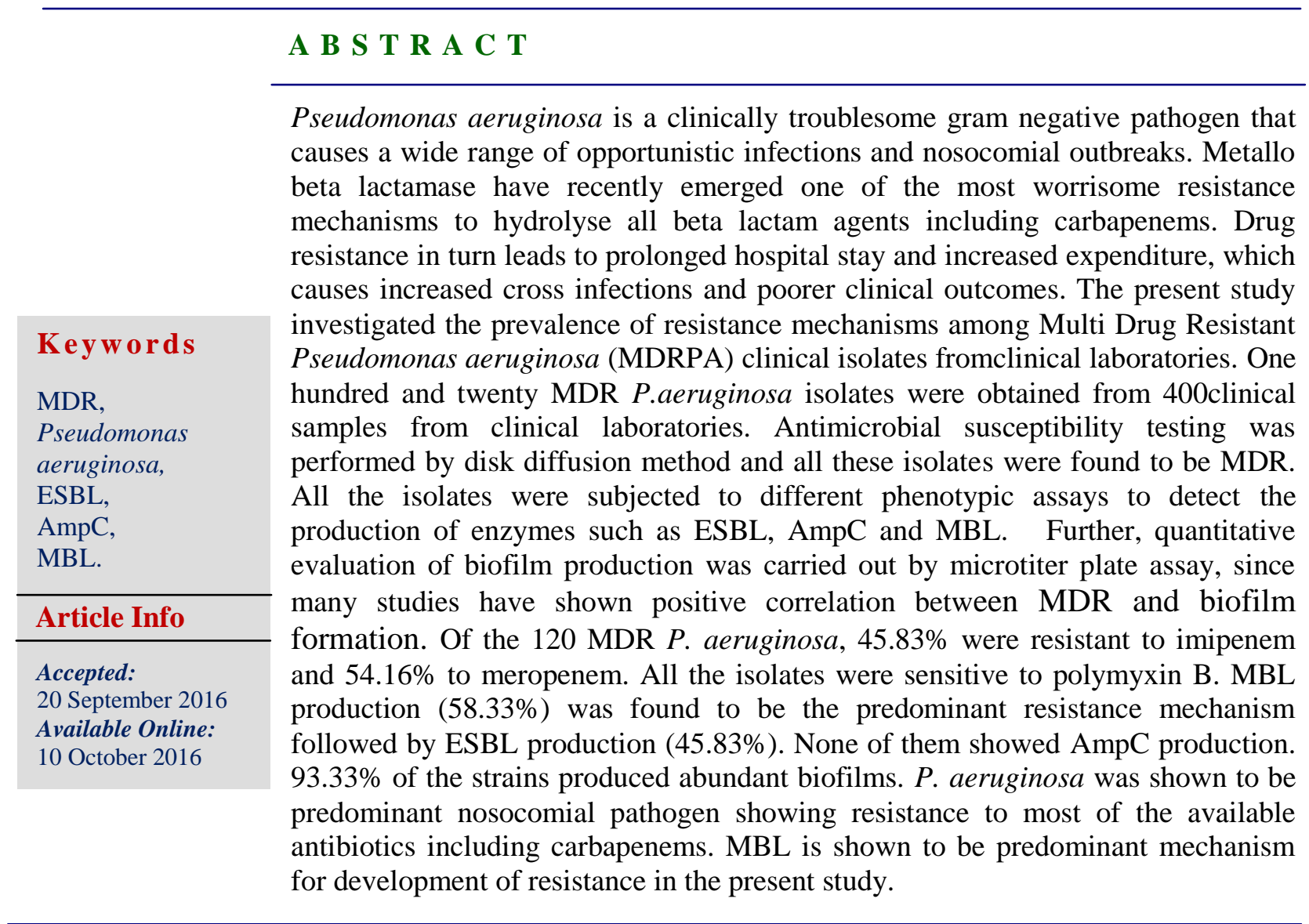

\section{Introduction}

Pseudomonas aeruginosa is a gram negative, non sporing, motile, aerobic bacilli cause of serious wound and surgical infections (Doggett, 1979). Infection caused by Pseudomonas aeruginosa is frequent amongst Hospital Acquired Infections
(HAI). Further, acquired drug resistance is common in nosocomial isolates of Pseudomonas spp (Hemalatha et al., 2005). The mechanism of resistance to beta lactam antibiotics includes, the production of beta lactamase, reduced outer membrane 
permeability, the altered affinity of target penicillin binding proteins, plasmid mediated resistance involving modifying enzymes (Cristina Lagatolla et al., 2004).

Metallo beta lactamases are class B beta lactamases. These require zinc or another heavy metal for their catalytic activity and their activities are inhibited by metal chelating agent such as EDTA and thiol based compounds (Johann et al., 2007). Acquired resistance is also reported by the production of plasmid mediated AmpC beta $(\beta)$ - lactamase, Extended Spectrum Beta $(\beta)$-Lactamase (ESBL) and metallo beta $(\beta)$-lactamase (MBL) enzymes (Supriya Upadhyay et al., 2010). Carbapenems are often used as antibiotics of last resort for treating infections due to multi-drug resistant Gram-negative bacilli as they are stable against ESBL and AmpC $\beta$-lactamase (Tanzinah Nasrin et al., 2010). However, Acquired MBL in Pseudomonas spp have recently emerged as one of the most worrisome resistance mechanism because of their capacity to hydrolyze all beta $(\beta)$ lactam antibiotics including penicillins, cephalosporins and carbapenems, with the exception of aztreonam (Mehul et al., 2011). Based on molecular studies, cabapenemhydrolyzing enzymes are classified into four groups A, B, C and D. The MBLs belong to group $\mathrm{B}$. The genes responsible for MBL production may chromosomally or plasmid mediated and hence poses a threat for spread of resistance by gene transfer among the Gram-negative bacteria (Bush, 1998). MBL producing Gram negative bacilli, specially Pseudomonas spp, have been increasingly reported in Asia, Europe, Latin American and the United States.

The appearance of MBL genes and their spread among bacterial pathogens is a matter of concern with regard to the future antimicrobial chemotherapy (Walsh et al.,
2005). Further, due to increase occurrence and types of these multiple $\beta$ lactamase enzymes, early detection is crucial, the benefits of which includes implementation of proper / optimal antibiotic therapy particularly in critically ill and hospitalized patients, infection control policy and to control the spread of resistance (Richet et $a l ., 2001)$. Thus, our study was undertaken to detect the MBL (metallo- $\beta$-lactamase) positive isolates of Pseudomonas aeruginosa from different clinical samplesfrom clinical laboratories.

\section{Materials and Methods}

The present study was carried out on P.aeruginosa obtained from various clinical samples from clinical laboratories during the period March 2015 to February 2016. One hundred and twenty MDR $P$. aeruginosa isolates were obtained from 400 clinical samples. The samples from which the strains were isolated include blood, Pus, Urine, Broncho alveolar lavage and endotracheal aspirates and tissues. Repeat isolates were excluded from the study. All the samples were processed for isolation and antibiotic sensitivity. Isolation of organism was done by streaking the samples on MacConkey's agar and Blood agar plates. Further identification was done by gram staining (gram negative bacilli), catalase (positive), oxidase(positive), and pigment production (positive), hanging drop preparation(motile) was done. ATCC P. aeruginosa 27853 strain was used as quality control reference strain for all experiments with satisfactory results.

Antibiotic sensitivity testing method was performed by Kirby Bauer method. Antibiotics included in the study are piperacillin $(75 \mu \mathrm{g})$, Piperacillin/Tazobactam (100/10 $\mu \mathrm{g}), \quad$ Ceftazidime $\quad(30 \mu \mathrm{g})$, Cefaperazone $(75 \mu \mathrm{g})$, Ceftriaxone $(30 \mu \mathrm{g})$, Imipenem $(10 \mu \mathrm{g})$, Meropenem(10 $\mu \mathrm{g})$, 
Gentamicin $(10 \mu \mathrm{g}), \quad$ Amikacin $(30 \mu \mathrm{g})$, Norfloxacin $(10 \mu \mathrm{g}), \quad$ Ciprofloxacin $(5 \mu \mathrm{g})$, Cefaperazone/Sulbactam $\quad(75 / 25 \mu \mathrm{g})$, Tobramycin $(10 \mu \mathrm{g})$, Netilmicin $(30 \mu \mathrm{g})$, Polymyxin B (300U), gatifloxacin (5ug).

All the MDR $P$. aeruginosa were subjected to different phenotypic assays to detect the production of enzymes such as ESBL, AmpC and MBL, which are implicated for causing multiple drug resistance. Phenotypic confirmatory test for ESBL production was performed by placing ceftazidime $(30 \mu \mathrm{g})$ and ceftazidime + clavulanic acid disc. Detection of Metallo- $\beta$-lactamases production test was carried out by combined disc diffusion test by placing 2 imipenem discs one with $0.5 \mathrm{M}$ EDTA and the other plain are placed on the surface of the agar plate approximately $30 \mathrm{~mm}$ apart. $\mathrm{AmpC}$ detection was done using AmpC discs method.

Microtitre plate assay for Biofilm production since many studies have shown positive correlation between biofilm and multiple drug resistance, quantitative evaluation of biofilm production by $P$. aeruginosa isolates was carried out by microtiter plate assay. Biofilm negative E. coli isolate from our collection and ATCC $P$. aeruginosa 27853 were used as negative and positive controls respectively. Based on the OD values, the extent of biofilm formed by the clinical isolates were classified as follows,

- $\mathrm{OD} \leq \mathrm{ODc}$ - Non adherent

- $\mathrm{ODc}<\mathrm{OD} \leq 2 \times$ ODc- Weakly adherent

- $2 \times$ ODc $<$ OD $4 \times$ ODc- Moderately adherent

- $4 \times \mathrm{ODc}<\mathrm{OD}-$ Strongly adherent (16).

\section{Results and Discussion}

A total of 120 clinical isolates of MDR Pseudomonas aeruginosa identified from Clinical Microbiology laboratories for a period of one year were included in this study. Among these 0 ne hundred and twenty isolates from MDRPA infection, male were found to be more predominant $71(59.16 \%)$ than females $49(40.83 \%) .40 \%(48)$ between 41 to 60 years of age group, 25\%(30) between 21 to 40 years of age group, same number of cases $30(25 \%)$ among the age group above 61 years and $12(10 \%)$ in less than 20 years of age. MDR Pseudomonas aeruginosa infection were mainly from RTA injuries $49(40.8 \%)$, Sepsis $9(7.5 \%)$, cellulitis 3(2.5\%), COPD 6(5.83\%), CVA \& Pneumonia 14(11.6\%), throat infection $4(3.3 \%)$, non-healing wound 4(3.3\%), infectious bedsore 13(10.8\%), DM foot $2(1.66 \%)$, fever $1(0.83 \%)$, UTI $1(0.83 \%)$, RT foot ulcer $1(0.83 \%)$, hip dislocation 7 $(5.83 \%), 5(4.1 \%)$ infectious sutures and severe LR 4(3.3\%).

Different types of specimens were, endotracheal (ET) aspirates $48(40 \%)$, wound swab 42(35\%), followed by blood 20(16.66\%), Broncho alveolar lavage (BAL)4(3.3\%), urine 3(2.5\%) and tissue $3(2.5 \%)$ respectively. Table (1)

All one hundred and twenty cases of MDRPA isolates were found to be $100 \%$ sensitive to Polymyxin B. They showed highest resistance towards Ciprofloxacin $93.33 \%(112)$ and tobramycin $84.16 \%(101)$, ninety eight isolates $(81.66 \%)$ resistance to Ceftriaxone, eighty percent (96) resistance to ceftazidime, $79.16 \%$ (95) resistance togatifloxacin, $76.66 \%$ (92) resistance towards Cefoperazone, $72.5 \%$ (87) to Cefoperazone/Sulbactam and 70.83\% (85) towards gentamycin. Seventy four isolates (61.66\%) showed resistance to Piperacillin, 
$55 \%$ (66) were resistant to Netilmicin and $65(54.16 \%)$ resistant to meropenem $52.5 \%$ (63) resistance were noticed for Amikacin, $59(49.1 \%)$ isolates shows resistant towards Piperacillin/Tazobactam. These strains showed resistance of $45.83 \%$ (55) towards Imipenem and none of them were resistant to Polymyxin B. Among these one hundred and twenty MDRPA strains table (2).

MIC for meropenem ranged from $0.5 \mu \mathrm{g} / \mathrm{ml}$ to $>64 \mu \mathrm{g} / \mathrm{ml}$. Sixty five of one hundred and twenty $(54.16 \%)$ isolates of MDRPA were found to be resistant to meropenem. The isolates were categorized resistant if the MIC value was more than $8 \mu \mathrm{g} / \mathrm{ml}$. fifty four (45\%) isolates showed sensitive MIC value $(\leq 4 \mu \mathrm{g} / \mathrm{ml})$ and $1(0.8 \% \mathrm{~b})$ isolates showed intermediate MIC value $(8 \mu \mathrm{g} / \mathrm{ml})$. All the isolates showed lower MIC of $0.5 \mu \mathrm{g} / \mathrm{ml}$ to $1 \mu \mathrm{g} / \mathrm{ml}$ for polymyxin B (Break point MIC for Pseudomonas aeruginosa $\leq 2 \mu \mathrm{g} / \mathrm{ml}$ to $\geq 8 \mu \mathrm{g} / \mathrm{ml}$ ) and none of these isolates showed resistant or intermediate MIC values.

Seventy isolates (58.33\%) were found to produce MBL and only fifty five (46\%) isolates showed ESBL production, none of the isolates showed AmpC production. Twenty $(16.66 \%)$ isolates were found to be negative for phenotypic production of all $\beta$ lactamases and 25(20.83\%) shows both ESBL and MBL production (table-3). Almost all isolates $(93.33 \%)$ from our collection were biofilm producers, wherein $75 \%$ of the isolates were strongly adherent, $8 \%$ moderately adherent and $11 \%$ weakly adherent. The percentage of non-adherent cells or biofilm negative isolates was found to be a meager of $7 \%$.

Pseudomonas aeruginosa is the leading cause of nosocomial infections, including pneumonia, urinary tract infections, and bacteremia. P.aeruginosa exhibits intrinsic resistance to several antimicrobial agents. However, acquired resistance to anti- pseudomonal $\beta$-lactams such as ticarcillin, piperacillin, ceftazidime, cefepime, aztreonam and carbapenems considered as deterrent weapon that can be a major challenge in managing MDRPA infections, especially while it is associated with coresistance with other classes of drugs namely aminoglycosides andfluoroquinolones. Several mechanisms can contribute to the acquired $\beta$-lactam resistance in P.aeruginosa, that includes production of $\beta$-lactamases, the upregulation of efflux systems, and decreased outer membrane permeability. With respect to $\beta$ lactamase production, acquired extendedspectrum $\beta$-lactamases (ESBL) and Metallo$\beta$-lactamases are the predominant emerging resistance mechanisms in P.aeruginosa. The present study aimed at elucidating major resistance mechanisms in MDRPA infections. We were encountered with more isolates producing MBL when compared to other mechanisms in MDRPA infections.

The prevalence of MDR $P$. aeruginosa was found to be $33.33 \%$ in our investigation, which is lesser in accordance with a recent study from India that showed the predominance rate of $44 \%$. However, comparatively lesser prevalence of MDRPA $(26.7 \%)$ responsible for burn wound infections in Iran (Antonopoulo et al., 2007). Likewise, one more investigation from India reported 22\% MDRPA and 4\% Pandrug resistant $P$. aeruginosa, wherein, we recorded a higher level of MDRPA incidence. Factors such as age and sex among MDRPA infection were found to have significant association with MDRPA, wherein the incidence was more among the age groups between 41 and $60(40 \%)$ and males being predominant $(59.16 \%)$ which is the likely case in earlier reports. Possibly, it may be due to high incidence of road traffic accidents among males, leading to hospitalization thereby high incidence of $P$. 
aeruginosa infection through catheterization. Earlier investigations have reported the major source of MDRPA to be sputum, tracheostomy specimen, pus, respiratory tract, surgical sites and endotracheal aspirate (Aggarwal et al., 2008; Shanthi et al., 2009). In the present study, the major source of MDRPA was found to be endotracheal aspirate (40\%),followed bywound swabs (35\%), implying that wound infections and respiratory tract infections are most significant infections caused by MDRPA in most of the hospitals. The major risk factors were prolonged hospitalization followed by patients on Foleys catheter. Foot infections and surgical site infections were found to be common source of MDRPA among the diabetic patients. MDRPA isolates showed markedly high-level resistance towards ciprofloxacin (93.33\%), tobramycin $(84.16 \%)$, followed by ceftriaxone $(81.66 \%)$, ceftazidime $(80 \%)$, gatifloxacin (79.16\%), cefoperazone (76.66\%), cefoperazone/ sulbactam (72.5\%), gentamicin, (70.83\%) piperacillin (61.66\%). Sixty three isolates (52.5\%) showed resistance to amikacin and $49.1 \%$ resistance was noticed for piperacillin/ tazobactam combination which is likely in the very recently published reports (Priyanka et al., 2016). Among carbapenems, imipenem and meropenem resistance was observed to be fifty five isolates $(45.83 \%)$ and sixty five isolates $(54.16 \%)$ respectively which is likely case in recently published reports (Samira et al., 2014). None of the isolates were resistant to polymyxin B. MIC for meropenem ranged from $0.5 \mu \mathrm{g} / \mathrm{ml}$ to $>64 \mu \mathrm{g} / \mathrm{ml}$. sixty five of $120(54.16 \%)$ isolates of MDRPA were found to be resistant to meropenem. The isolates were categorized resistant if the MIC value was more than $8 \mu \mathrm{g} / \mathrm{ml}$. fifty four $(45 \%)$ isolates showed sensitive MIC value $(\leq 4 \mu \mathrm{g} / \mathrm{ml})$ and $1(0.8 \%)$ isolates showed intermediate MIC value $(8 \mu \mathrm{g} / \mathrm{ml})$. All the isolates showed lower MIC of $0.5 \mu \mathrm{g} / \mathrm{ml}$ to $1 \mu \mathrm{g} / \mathrm{ml}$ for polymyxin B (Break point MIC for Pseudomonas aeruginosa $\leq 2 \mu \mathrm{g} / \mathrm{ml}$ to $\geq 8 \mu \mathrm{g} / \mathrm{ml}$ ) and none of these isolates showed resistant or intermediate MIC values.

Among various mechanisms of resistance, MBL and ESBL enzymes were found to be more effective and the incidence of MBL production in P.aeruginosa has been reported to be $10-30 \%$ from different clinical setups in India (Navaneeth et al., 2002). Previous studies have shown a incidence of MBL (47\%), AmpC (50\%) and ESBL (13.3\%) among the MDRPA isolates tested (Morten et al., 2001). In another study shows MBL (36\%) among MDRPA were isolated. In our study, we have observed a little high prevalence of MBL (58.33\%), ESBL (45.83\%) producers and $20.83 \%$ of MDRPA isolates were found to produce both MBL and ESBL, which appears to be significant.

Table.1

\begin{tabular}{|l|l|}
\hline \multicolumn{2}{|c|}{$\begin{array}{c}\text { Table.1 Kind of samples and } \\
\text { their numbers }\end{array}$} \\
\hline SAMPLES & TOTAL NO. \\
\hline ET aspirate & $48(40 \%)$ \\
\hline BAL & $4(3.3 \%)$ \\
\hline Blood & $20(16.66 \%)$ \\
\hline Wound swab & $42(35 \%)$ \\
\hline Tissue & $3(2.5 \%)$ \\
\hline Urine & $3(2.5 \%)$ \\
\hline
\end{tabular}


Table.2

\begin{tabular}{|l|l|l|l|}
\hline \multicolumn{5}{|c|}{ Table.2 Antibiotic resistance among isolates of MDR P.aeruginosa } \\
\hline ANTIBIOTICS & RESISTANT & SENSITIVE & INTERMEDIATE \\
\hline piperacillin (75ug) & $74(61.66 \%)$ & $46(38.33 \%)$ & $0(0 \%)$ \\
\hline $\begin{array}{l}\text { piperacillin-tazobactam } \\
\text { (100/10ug) }\end{array}$ & $59(49.1 \%)$ & $54(45 \%)$ & $7(5.83 \%)$ \\
\hline ceftazidime(30ug) & $96(80 \%)$ & $15(12.5 \%)$ & $9(7.5 \%)$ \\
\hline cefoperazone(75ug) & $92(76.66 \%)$ & $9(7.5 \%)$ & $19(15.83 \%)$ \\
\hline ceftriaxone(30ug) & $98(81.66 \%)$ & $9(7.5 \%)$ & $13(10.83 \%)$ \\
\hline imipenem(10ug) & $55(45.83 \%)$ & $65(54.16 \%)$ & $0(0 \%)$ \\
\hline meropenem(10ug) & $65(54.16 \%)$ & $54(45 \%)$ & $1(0.8 \%)$ \\
\hline gentamicin(10ug) & $85(70.83 \%)$ & $35(29.16 \%)$ & $0(0 \%)$ \\
\hline amikacin(30ug) & $63(52.5 \%)$ & $44(36.66 \%)$ & $13(10.83 \%)$ \\
\hline ciprofloxacin(5ug) & 112 & $4(3.3 \%)$ & $4(3.3 \%)$ \\
\hline cefoperazone-sulbactam(75/25ug) & $87(93.33 \%)$ & & \\
\hline tobramycin(10ug) & 101 & $11(9.16 \%)$ & $22(18.33 \%)$ \\
\hline netilmicin(30ug) & $(84.16 \%)$ & $19(15.83 \%)$ & $0(0 \%)$ \\
\hline gatifloxacin(5ug) & $66(55 \%)$ & $54(45 \%)$ & $0(0 \%)$ \\
\hline PolymyxinB(300U) & $95(79.16 \%)$ & $18(15 \%)$ & $7(5.83 \%)$ \\
\hline & $00(0 \%)$ & $120(100 \%)$ & $0(0 \%)$ \\
\hline
\end{tabular}

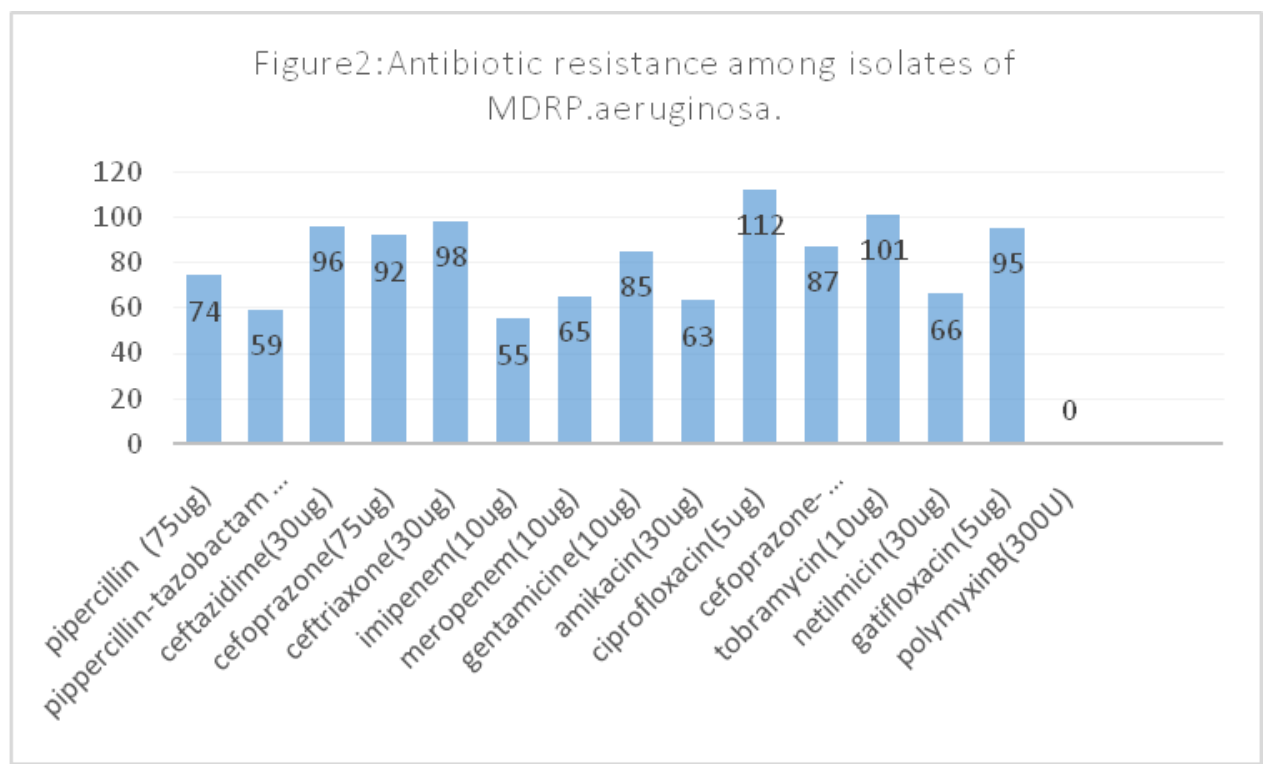




\begin{tabular}{|l|l|l|}
\hline \multicolumn{3}{|c|}{$\begin{array}{c}\text { Table.3. Prevalence of various Beta lactamases } \\
\text { mediated resistance mechanisms among the clinical } \\
\text { isolates of MDRPA on phenotypic tests }\end{array}$} \\
\hline & NEGATIVE & POSITIVE \\
\hline MBL & $50(41.66 \%)$ & $70(58.33 \%)$ \\
\hline ESBL & $65(54.16 \%)$ & $55(45.83 \%)$ \\
\hline Both MBL \& ESBL & $20(16.66 \%)$ & $25(20.83 \%)$ \\
\hline Biofilms & $8(6.66 \%)$ & $112(93.33)$ \\
\hline AmpC & $120(100 \%)$ & 00 \\
\hline
\end{tabular}

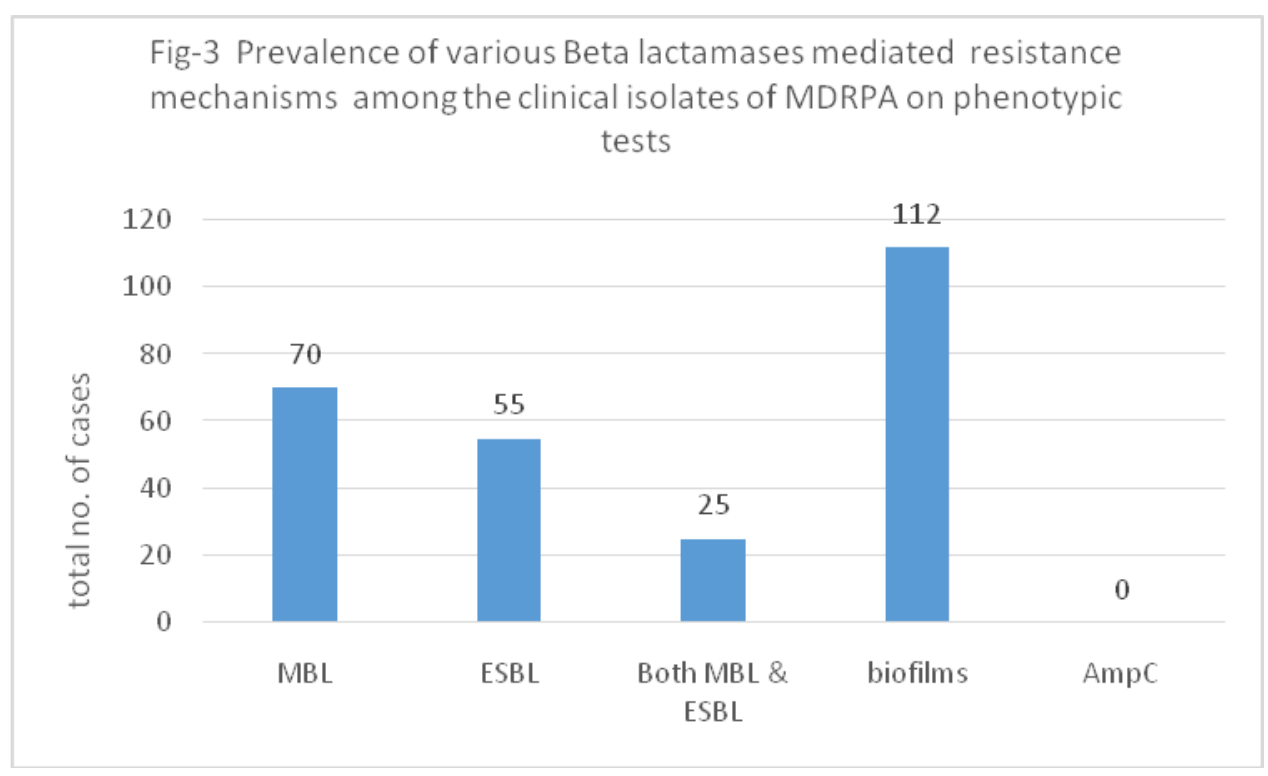

Around $16.66 \%$ of isolates did not show any of the mechanisms studied which might follow altogether different resistance mechanisms like formation of biofilms and/or cell wall permeability defects and efflux pump mechanisms. High percentage of biofilm producers were observed in our study, which may be due to the increase number of MDRPA isolates encountered. Morten Hentzer et al., earlier reported the strong correlation between biofilm formation and multiple drug resistance in Gram negative pathogens. Thus, biofilm formation appears to be one of the mechanisms among these strains to develop multi drug resistance, which is evident from the earlier reports from India.
In conclusion, in summary, MDR $P$. aeruginosa is a notable cause of hospital acquired infections and known to cause a wide spectrum of life threatening diseases..MBL production is the major cause for resistance to carbapenem group of antibiotics which are considered to be effective drugs for treatment of infections caused by P.aeruginosa. These organisms are resistant to almost all commonly available antibiotics with limited treatment options. Forty sixpercent of isolates showed resistance to imipenem and $54 \%$ to meropenem, which is an "alarming sign", since carbepenems were the present drug of choice. Furthermore, $93 \%$ of isolates had the ability to form biofilm that might aid in the 
persistence of MDRPA thereby imparts resistance. Routine detection of MBLs will ensure optimal patient care and timely introduction of appropriate infection control procedure.

\section{Declaration of Interest}

The authors report no conflicts of interest. The authors alone are responsible for the content and writing of the paper.

\section{Statistical analysis}

All the data were entered in Microsoft Excel sheet and the results were analyzed by SPSS software. (IBM, USA)

\section{References}

Aggarwal, R., Chaudhary, U., Bala, K. 2008. Detection of extended-spectrum betalactamase in Pseudomonas aeruginosa. Indian J. Pathol. Microbiol., 51(2): 48-51.

Ambler, R.P. 1998. The structure of betalactamases. Philos Trans R Soc London Bio Sci 1980; 289: 321-31.

Antonopoulo, A., Raftogiannis, M., Giamarellos-Bourboulis, E.J. 2007. Early apoptosis of blood monocytes is a determinant of survival in experimental sepsis by multidrugresistant Pseudomonas aeruginosa. Clin. Exp. Immunol., 149(1): 103-8.

Bush K. Metallo $\beta$-lactamase: a class apart. Clin. Infect. Dis., 27 (Suppl 1): S4853.

Cristina Lagatolla, et al. 2004. Endemic carbapenem resistant Pseudomonas aeruginosa with acquired metallo beta lactamase determinants in European hospital. Emerg. Infect. Dis., Vol. 10(3): 535-538.
Davies, J.C., Bilton, D. 2009. Biofims and reistance in cystic fibrosis. Respir. Care, 54: 628-640.

Doggett, R.G. 1979. Microbiology of Pseudomonas aeruginosa. In: Dogett R.G, ed. Pseudomonas aeruginosa. Clinical manifestations of infection and current therapy. New York Academic press, 1-8.

Hemalatha, V., Uma Sekar, Vijay Lakshmikamath. 2005. Detection of metallobeta lactamase producing Pseudomonas aeruginosa in hospitalized patients. Indian J. Med. Res., 148-152.

Jayakumar, S., Appalaraju, B. 2007. Prevalence of multi and pan drug resistant Pseudomonas aeruginosa with respect to ESBL and MBL in a tertiary care hospital. Indian J. Pathol. Microbiol., 50(4): 922-5.

Johann, D.D., Pitout, et al. 2007. Molecular epidemiology of metallo beta lactamase producing Pseudomonas aeruginosa in the Calgary health region: emergence of VIM-2 producing isolates. J. Clin. Microbiol., 2: 294-298.

Kiran Ruhil, Bharti Arora, Himanshu Adlakha. 2009. Pseudomonas aeruginosa isolation of Post-operative wound in a referral hospital in Haryana, India. J. Infect. Dis. Antimicrob. Agents, 26: 43-8.

Manchanda, V., Singh, N.P. 2008. Occurrence and detection of AmpC blactamase, among Gram negative clinical isolates using a modified threedimensional test at Guru Teg Bahadur Hospital, Delhi, India. $J$. Antimicrob. Chemother., 51: 415418.

Mehul, S., Chaudhari, Tanuja, B., Javdekar, GovindNinama, Neelam Pandya, JivrajDamor. 2011. A Study of Metallo-beta-lactamase producing Pseudomonas aeruginosa in clinical 
samples of SSG Hospital. National $J$. Med. Res., 1(2): 60-63.

Morten Hentzer, L., Gail, M., Teitzel, Grant, J., Balzer, Arne Heydorn, L., Søren Molin, L., Michael Givskov, L., Matthew, R., Parsek. 2001. Alginate Overproduction Affects Pseudomonas aeruginosa. Biofilm Structure and Function. J. Bacteriol., 5395-5401.

Nagaveni, S., Rajeshwari, H., Ajay Kumar oli, S.A., Patil and R., Kelmani Chandrakanth. 2010. Evaluation of Biofilm forming ability of the multidrug resistant Pseudomonas aeruginosa. The Bioscan, 5(4): 563$56,222-4$.

Navaneeth, B.V., Sridaran, D., Sahav, D., Belwadi, M.R. 2002. A preliminary study on MBL producing Pseudomonas aeruginosa in hospitalized patient. Indian J. Med. Res., 116: 264-7.

Poirel, L., Naas, T., Nicholas, D., Collet, L., Bellais, S., Cavallo, J.D., et al. 2000. Characterization of VIM-2, a Carbapenem-hydrolyzing metallobeta-lactamase and its plasmid-and integron-born gene from a Pseudomonas aeruginosa clinical isolate in France. Antimicrob. Agents Chemother., 44: 891-97.

Priyanka Meel, Manju Meel, B. Kumar. 2016. Study of Antimicrobial Susceptibility of Pseudomonas aeruginosa Isolated from Wound infections in Indian Population. Int. J. Scientific Res., 2277-8179.

Richet, H.M., Mohammed, J., McDonald, L.C., Jarvis, W.R. 2001. Building communication networks: international network for the study and prevention of emerging antimicrobial resistance. Emerg. Infect. Dis., 7: 31922.

Samira Aghamiri, Nour Amirmozafari, Jalil Fallah Mehrabadi, Babak Fouladtan, and Hossein Samadi Kafil. 2014. Antibiotic Resistance Pattern and Evaluation of Metallo-Beta Lactamase Genes Including bla-IMP and bla-VIM Types in Pseudomonas aeruginosa Isolated from Patients in Tehran Hospitals. Hindawi Publishing Corporation ISRN, Microbiol., 10.1155-941507.

Shankar, E.M., Mohan, V., Premalatha, G. 2005. Bacterial etiology of diabetic foot infections in South India. Eur. J. Intern. Med., 16(8): 567-70.

Shanthi, M., Sekar, U. 2009. Multi-drug resistant Pseudomonas aeruginosa and Acinetobacter baumannii infections among hospitalized patients: risk factors and outcomes. J. Assoc. physicians India, 57: 636-645.

Shashikala, Kanungo, R., Srinivasan, S., Devi, S. 2006. Emerging resistance to carbapenem in hospital acquired Pseudomonas infection: Indian J. Pharmacol., 38: 287-8.

Stepanovic, S., Vukovic, D., Dakic, I., Savic, B. 2000. A modifiedmicrotiterplate test for quantification of staphylococcal biofilm formation. $J$. Microbiol. Methods, 40: 175-179.

Supriya Upadhyay, Malay Ranjan Sen, Amitabha Bhattacharjee. 2010. Presence of different betalactamase classes among clinical isolates of Pseudomonas aeruginosa expressing AmpC beta-lactamase enzyme. $J$. Infect. Dev. Ctries, 4(4): 239-242.

Taneja, N., Aharwal, S.M., Sharma, M. 2003. Imipenem resistance in nonfermenters causing nosocomial urinary tract infection. Indian J. Med. Sci., 57: 294.

TanzinahNasrin, Md. SharifulAlamJilani, Lovely Barai, J. AshrafulHaq. 2010. Metallo- $\beta$ Lactamase Producing Pseudomonas species in a Tertiary Care Hospital of Dhaka City, 
Bangladesh J. Med. Microbiol., 04(01): 4345.

Toniolo, A., Endimiani, A., Luzzaro, F. 2006. Microbiology of postoperative infections. Surg. Infect., 7 suppl 2: S1316.

Vasundhara Devi, P., P. Sreenivasulu Reddy and Maria Sindhura John. 2015. Prevalence of Metallo- -Lactamases
Producing Pseudomonas aeruginosa among the Clinical isolates: A study from tertiary care hospital. Int. J. Curr. Microbiol. Appl. Sci., 955-961. Walsh, T.R., Toleman, M.A., Poirel, L., Nordmann, P. 2005. Metallo- $\beta$ lactamase: the Quiet before the Storm? Clin. Microbiol. Rev., 18: 306-25.

\section{How to cite this article:}

Mohammed Ansar Qureshi and Rakesh Kumar Bhatnagar. 2016. Phenotypic Characterization of ESBL, AmpC and MBL Producers among the Clinical Isolates of Multidrug Resistant Pseudomonas aeruginosa. Int.J.Curr.Microbiol.App.Sci. 5(10): 749-758. doi: http://dx.doi.org/10.20546/ijcmas.2016.510.081 